\title{
Una herramienta para el análisis del nivel de comprensión del modelo de materia de los alumnos de $4^{\circ}$ de ESO
}

\author{
María Ángeles Moltó Palomares \\ Institut Sant Quirze del Vallès Departament d'Educació Generalitat de Catalunya. España. \\ angelsmolto@gmail.com \\ María Isabel Hernández Rodríguez (D) , Roser Pintó Casulleras \\ Departament de Didàctica de la Matemática i de les Ciències Experimentals Universitat Autònoma de \\ Barcelona. España. \\ marisabernandez@uab.cat,roserpinto@uab.cat
}

[Recibido: 27 diciembre 2019. Revisado: 31 mayo 2020. Aceptado: 15 octubre 2020]

Resumen: En este estudio, se ha desarrollado una herramienta analítica para caracterizar las concepciones de los estudiantes de $4^{\circ}$ de ESO sobre la materia. Se ha considerado que, en este nivel de instrucción, el modelo científico escolar de materia que se utiliza debería ser un modelo avanzado que difiera del modelo básico de materia en la distinción de las partículas entre átomos, moléculas e iones. Este modelo avanzado podría permitir que los estudiantes hicieran interpretaciones bien fundamentadas de los cambios físicos y químicos más importantes de la materia. La herramienta analítica aquí presentada incluye cuatro dimensiones (Conformación, Dinámica, Interacciones y Diversidad). Estas dimensiones abarcan un conjunto de categorías que permiten caracterizar las concepciones de los estudiantes sobre la materia. Para construir esta herramienta analítica definimos un conjunto de dimensiones y categorías inspiradas en las ya establecidas por Talanquer (2009), y que emergen del análisis de las respuestas de los estudiantes cuando representan la materia a nivel submicroscópico.

Palabras clave: modelo de materia básico y avanzado; enseñanza obligatoria

An analytical tool to explore 15-16 year old students' understanding of an advanced model of matter

Abstract: In this study, an analytical tool has been developed to characterise 15-16 year old students' conceptions about matter. It has been considered that at this instructional level the school scientific model of matter that is used should be an advanced model that differs from a basic model of matter in the distinction of particles as atoms, molecules and ions. This advanced model could allow students to provide well founded interpretations of most important physical and chemical changes of matter. The analytical tool here reported includes four dimensions (Conformation, Dynamics, Interactions and Diversity). These dimensions encompass a set of categories that allow characterising students' conceptions of matter. In order to build the analytical tool here reported, we defined a set of dimensions and categories which are drawn upon the ones established by Talanquer (2009) but also emerged from the analysis of students' answers when representing matter at a submicroscopic level.

Keywords: basic and advanced model of matter, secondary teaching

Para citar este artículo: Moltó Palomares M.A., Hernández Rodríguez M.I., Pintó Casulleras R. (2021) Una herramienta para el análisis del nivel de comprensión del modelo de materia de los alumnos de $4^{\circ}$ de ESO. Revista Eureka sobre Enseñanza y Divulgación de las Ciencias 18(1), $1104 . \quad$ doi: 10.25267/Rev_Eureka_ensen_divulg_cienc.2021.v18.11.1104

\section{Introducción y antecedentes}

\section{Justificación de la investigación y objetivos}

Fenómenos tan cotidianos como la fusión, la disolución, la ebullición y la transformación de las sustancias, que para los expertos son fáciles de interpretar a partir del modelo científico de materia, pueden plantear en su interpretación y explicación grandes dificultades a los 
estudiantes de secundaria (Barke, Hazari y Yitbarek 2009). Por ejemplo, los estudiantes de secundaria muestran dificultades importantes en la interpretación de por qué diferentes sustancias (por ejemplo, sal y azúcar) se comportan de manera diferente en relación con la conducción eléctrica cuando se disuelven en agua (Moltó y Pintó 2013). Otro ejemplo de dificultad común es la dificultad para caracterizar determinados fenómenos como cambio físico o un cambio químico después de observar la aparición de burbujas, ya que algunos estudiantes interpretan este hecho como un cambio de estado de una sustancia mientras que otros lo interpretan como una descomposición/composición de sustancias. Las dificultades de estos estudiantes para interpretar el comportamiento y cambio de la materia están relacionadas con sus concepciones sobre la estructura interna de la materia, es decir, sobre las partículas submicroscópicas que forman las sustancias y las uniones entre ellas. Una adecuada comprensión de estos fenómenos puede permitirles tomar decisiones informadas en asuntos sociocientíficos relevantes o controvertidos, como los relacionados con el uso de combustibles fósiles, el reciclaje de materiales, los riesgos de los materiales nanotecnológicos, los límites de la electrónica de silicio, entre otros. En este sentido, nuestro objetivo final es ayudar a nuestros estudiantes a convertirse en ciudadanos científicamente alfabetizados y responsables que puedan tomar decisiones basadas en la evidencia.

En las últimas cuatro décadas, se han realizado numerosos estudios que han descrito los modelos de materia de los estudiantes (Hadenfeldt, Liu y Neumann 2014; Liu y Lesniak 2006 y 2005; Benarroch 2000a; Johnson, 1998; Renström, Andersson y Marton 1990; Andersson 1990).

Por otro lado también se han propuesto diferentes progresiones de aprendizaje sobre la materia para diferentes niveles educativos (Johnson y Tymms 2011; Stevens, Delgado y Krajcik 2010; Smith, Wiser, Anderson y Krajcik 2006; Liu y Lesniak 2006 y 2005). Vemos pues que la investigación educativa en el dominio de la conceptualización de la materia ya ha proporcionado información sobre las dificultades de los estudiantes y sobre algunas vías para contribuir al desarrollo de un modelo corpuscular de la materia consistente con el científico. Sin embargo, no hay estudios específicos sobre cómo hacer que los estudiantes de secundaria avancen en la construcción de un modelo avanzado de materia desde la educación secundaria inferior hasta la educación secundaria superior ( $4^{\circ}$ de ESO). En este sentido, nuestro proyecto de investigación se centra en llenar este vacío.

El estudio de investigación presentado aquí tiene como objetivo caracterizar las concepciones sobre la materia de los estudiantes de $4^{\circ}$ de ESO para elaborar una herramienta analítica que permita explorar las concepciones de los estudiantes. Este instrumento de análisis define unas dimensiones y categorías que emergen del análisis de las producciones de los estudiantes que son las respuestas a las actividades de la secuencia de enseñanza aprendizaje (SEA) diseñada y está inspirado en el trabajo de Talanquer (2009).

Así nuestra pregunta de investigación es:

¿Cómo podemos caracterizar las concepciones de la materia de los estudiantes de $4^{\circ}$ de ESO en relación con un modelo avanzado de la materia?

\section{¿Cuál es el modelo científico de materia adecuado para los alumnos de secundaria superior?}

La materia es uno de los conceptos más centrales de la ciencia y, como tal, ha sido estudiada desde varias perspectivas y con diferentes objetivos. En este estudio, el modelo corpuscular de materia propuesto por la ciencia se considera derivado de los principios de la teoría cinético corpuscular de la materia (TCC). Para fines didácticos estos principios deben ser reconstruidos educativamente y definirse para la ciencia escolar (Duit, Gropengießer y Kattman 2005). En 
otras palabras, el modelo científico debe ser transpuesto didácticamente para dar lugar a un modelo científico escolar (Izquierdo-Aymerich y Adúriz-Bravo 2003).

Diferentes autores han transpuesto didácticamente la TCC para utilizarla en diferentes niveles educativos. Tradicionalmente, las afirmaciones de la TCC de la materia se han utilizado para justificar cambios físicos; mientras que la teoría atómica de Dalton junto con otras teorías (por ejemplo, teoría de enlaces) se han utilizado para explicar los cambios químicos (Stevens et al. 2010; Benarroch 2000b; Johnson 1998). Así, autores como De Vos y Verdonk (1996) formularon un conjunto de enunciados de la TCC para la educación primaria en ciencias. Stern y Ahlgren (2002) compartieron otro conjunto de enunciados de la teoría cinético molecular para la enseñanza secundaria. Wiser y Smith (2008) propusieron principios básicos para la teoría atómico-molecular que utilizan en los grados escolares K-8. Estos enunciados diferentes tienen en común que hacen referencia a la composición de la materia (la materia está formada por partículas), al movimiento de las partículas (las partículas están en continuo movimiento) y al tamaño de estas partículas (las partículas son extremadamente pequeñas y no se pueden ver) pero difieren en: a) la descripción de las partículas, b) la relación entre las partículas, y c) la justificación de los cambios que experimenta la materia en términos de partículas. Los enunciados utilizados por Stern y Ahlgren (2002) sólo justifican los cambios físicos, mientras que los utilizados por De Vos y Verdonk (1996) y Wiser y Smith (2008) justifican los cambios físicos y químicos. Observamos pues que no existe un conjunto de enunciados acordados por la comunidad educativa de un modelo científico escolar universal para explicar las transformaciones de la materia.

Nuestra propuesta didáctica para la enseñanza secundaria superior considera necesario ayudar a los estudiantes a pasar de un modelo escolar básico de materia, tal y como lo considera Johnson (1998), a un modelo escolar avanzado de materia consistente con el anterior y, como ya se dijo, con potencial para interpretar cambios tanto físicos como químicos. Con el modelo básico de materia, es decir, sin diferenciación entre moléculas, átomos e iones, los alumnos no podrían interpretar ciertas propiedades y comportamientos de las sustancias (elevada o baja temperatura de fusión o de ebullición, conducción eléctrica de las sustancias en estado líquido y en disolución, etc.). El modelo escolar básico de la materia se deriva tradicionalmente de la TCC, la cual, para establecer un modelo escolar avanzado de la materia se complementaría con la teoría atómica de Dalton y la teoría de enlaces, de modo que se puedan distinguir diferentes tipos de partículas (moléculas, átomos e iones). Otros autores han sugerido un enfoque similar para la instrucción de esta teoría argumentando su propuesta sobre una base histórica, es decir, sobre cómo la ciencia construyó esta teoría a lo largo de la historia (Harrison y Treagust 2002). Coincidimos con Talanquer (2018, p. 1003) cuando dice que: «un uso productivo de los modelos atómicos y de partículas para construir relaciones estructura-propiedad exige la identificación de las partículas relevantes en un sistema (por ejemplo, átomos, moléculas, iones) y la caracterización de su estructura e interacciones».

A la vista de lo expuesto anteriormente, para los propósitos de nuestra investigación, consideramos necesario especificar los enunciados que definen el modelo escolar avanzado de materia y, para ello, hemos reescrito los enunciados de la TCC para incluir las diversas partículas que forman los diferentes tipos de sustancias y la fuerza de las uniones entre partículas. Los enunciados que utilizamos en nuestro estudio se muestran en la tabla 1 . Estos enunciados son adaptaciones de los enunciados de la TCC de otros autores y han sido utilizados en sus investigaciones sobre las progresiones de aprendizaje de la materia y nos han inspirado en nuestra propuesta.

Como reconstrucción educativa que supone el diseño de la SEA se han considerado las ideas clave de la química actual (Chang 2002) y las necesidades educativas relacionadas con el tema 
identificadas a través de la práctica docente (Moltó y Pintó 2013) y a partir de la literatura del ámbito. Así consideramos que los dos enunciados destacados en negrita inciden en los aspectos más críticos para comprender a un nivel avanzado cómo se comporta la materia. No sólo disponemos de átomos o de partículas en general. Introducimos las moléculas como agrupaciones de átomos no metálicos y los iones como porciones del átomo con características diferentes (partículas cargadas). ${ }^{1}$

Tabla 1. Enunciados de la TCC para el estudio adaptados de De Vos y Verdonk (1998), Stern y Ahlgren (2002) y Wiser y Smith (2008).

\begin{tabular}{|c|c|}
\hline 1 & $\begin{array}{l}\text { Toda la materia esta hecha de átomos que son tan pequeños que no se pueden ver con un } \\
\text { microscopio }\end{array}$ \\
\hline 2 & $\begin{array}{l}\text { Los átomos se comportan como objetos duros, sólidos e inmutables. Sus dimensiones y su forma } \\
\text { son irrelevantes. En los dibujos, se pueden representar como círculos pequeños o puntos. }\end{array}$ \\
\hline 3 & Hay espacio vacío entre los átomos. \\
\hline 4 & Los átomos ocupan espacio, tienen masa y están en constante movimiento. \\
\hline 5 & Toda la materia que podemos encontrar en la Tierra está hecha de menos de 100 clases de átomos. \\
\hline 6 & $\begin{array}{l}\text { Cada clase de átomos tiene diferentes propiedades que incluyen su masa y la manera de combinarse } \\
\text { con otros átomos. }\end{array}$ \\
\hline 7 & $\begin{array}{l}\text { Los átomos se pueden combinar para formar moléculas o redes- un proceso que implica la } \\
\text { formación de enlaces químicos entre átomos. }\end{array}$ \\
\hline 8 & $\begin{array}{l}\text { Algunas sustancias (sustancias simples) están formadas por un tipo de átomos solamente. Otras } \\
\text { sustancias (compuestos) están formadas por grupos de átomos unidos entre sí. }\end{array}$ \\
\hline 9 & $\begin{array}{l}\text { Según el tipo de sustancia, podemos identificar las partículas como átomos o como iones } \\
\text { (estructuras gigantes de átomos o iones), o moléculas (agrupaciones de átomos). En las } \\
\text { estructuras gigantes, las fuerzas que unen los átomos o los iones entre sí son fuertes. En } \\
\text { las moléculas, las fuerzas que unen los átomos entre sí son fuertes, pero las fuerzas que } \\
\text { unen las moléculas entre sí son débiles. Los puntos de fusión y de ebullición de las } \\
\text { sustancias formadas por moléculas son bajos y los puntos de fusión y de ebullición de las } \\
\text { estructuras gigantes son altos. }\end{array}$ \\
\hline 10 & $\begin{array}{l}\text { Hay una relación directa entre la temperatura de la sustancia y la energía cinética media de sus } \\
\text { partículas. }\end{array}$ \\
\hline 11 & $\begin{array}{l}\text { Los cambios en la materia pueden ser cambios físicos, en los cuales las moléculas (en las } \\
\text { sustancias moleculares) o los átomos e iones (en las sustancias metálicas e iónicas) } \\
\text { cambian su organización y/o movimiento, pero permanecen intactas; y los cambios } \\
\text { químicos, en los cuales los átomos se recombinan (se unen o se separan) en nuevas } \\
\text { moléculas, pero los átomos permanecen intactos. }\end{array}$ \\
\hline
\end{tabular}

\section{¿Cómo conciben los estudiantes la materia?}

Después de hacer una revisión histórica de los estudios sobre el conocimiento de los estudiantes sobre la materia y su evolución a lo largo de la instrucción (Hadenfeldt et al 2014, Johnson 1998, Renström et al. 1990, Talanquer 2009) consideramos de particular relevancia el trabajo de caracterización realizado por Talanquer (2009). Este autor revisa varios estudios de investigación sobre el conocimiento de la materia por parte de los estudiantes a lo largo de la instrucción y establece obstáculos (supuestos implícitos) que los estudiantes deben superar para construir un modelo de materia consistente con el modelo conceptual propuesto por la

\footnotetext{
${ }^{1}$ Somos conscientes de que esta propuesta docente de un modelo avanzado de materia no es suficiente para interpretar ciertos cambios químicos como la oxidación y la reducción, pero permite justificar la mayoría de los cambios físicos y un número significativo de cambios químicos, la mayoría de los que se estudian en la escuela secundaria.
} 
ciencia. El estudio de Talanquer (2009) nos ha resultado muy útil como punto de partida para diseñar un instrumento que permita caracterizar las concepciones de los estudiantes en relación con el llamado modelo avanzado de la materia. Talanquer (2009) describe cuatro dimensiones para caracterizar las concepciones de la materia de los estudiantes. Cada una de estas dimensiones contiene diferentes categorías ordenadas de menor a mayor grado de sofisticación o cercanía al punto de vista científico.

Talanquer (2009) considera que el conocimiento de los estudiantes sobre la materia pasa de la idea de materia continua sin ninguna estructura subyacente, a través de la concepción de granularidad, que supone que la materia está formada por pequeñas piezas de sustancia o por partículas que están incrustadas en la sustancia, hasta la concepción de la discontinuidad de la materia. Esta concepción más avanzada considera que la materia está formada por partículas de una naturaleza distintiva, con diferentes propiedades de la sustancia misma, y estas partículas están separadas por vacío. Estas categorías se agrupan bajo la dimensión Estructura.

Dentro de la dimensión Propiedades, Talanquer (2009) indica que los estudiantes novatos asumen las sustancias como portadoras de las propiedades (Sustancialismo), los estudiantes intermedios consideran que los elementos químicos tienen algunas propiedades esenciales que pueden ser heredadas por las sustancias en las que están presentes (Elementalismo), y los estudiantes más avanzados entenderían que las propiedades de las sustancias emergen de las interacciones dinámicas de los electrones en los átomos, los átomos en las moléculas, y las moléculas en muestras macroscópicas de materiales (Emergencia).

La dimensión Dinámica tiene en cuenta el conocimiento de los estudiantes sobre el movimiento de las partículas. Así, los estudiantes novatos piensan que las partículas no se mueven (Estática). Otros estudiantes piensan que el movimiento de las partículas tiene una causa externa (Causal-dinámica). Los estudiantes que son más expertos en el tema consideran que las partículas están en movimiento continuo. Talanquer también distingue entre aquellos estudiantes que interpretan el movimiento de las partículas según el estado y la temperatura (Contingente-dinámica) y aquellos que consideran que el movimiento continuo de las partículas es una propiedad intrínseca (Intrínseca-dinámica).

Finalmente, la dimensión Interacción propuesta por Talanquer (2009) distingue entre estudiantes novatos que consideran que el contacto entre partículas es necesario para la interacción (Contacto interactivo), los estudiantes intermedios que consideran que existe una estrecha interacción que depende de factores como la temperatura y el estado de agregación de la sustancia (Contingente interactivo) y los estudiantes avanzados que consideran que esta interacción cercana es causada por un campo eléctrico creado por las propias partículas, es intrínseca y sólo depende de la distancia entre las partículas (Intrínsecamente interactiva).

En el presente estudio, nos hemos inspiramos en algunas de las dimensiones y categorías de Talanquer para interpretar las respuestas de los estudiantes, de manera que algunas de ellas se han reformulado para ajustarlas a nuestras observaciones y nuestro lenguaje y otras no se han incluido como consecuencia de la naturaleza de nuestro estudio en relación al de Talanquer. Así nosotros utilizamos el término Conformación porque según nuestra interpretación del término estructura, ésta viene determinada por la composición y los enlaces (es decir, las interacciones) entre componentes (es decir, partículas). Nuestra dimensión Dinámica solo considera dos de las categorías sugeridas por Talanquer (2009): Estática y Dinámica, ya que nuestros instrumentos para la recolección de datos no fueron diseñados para identificar las justificaciones de los estudiantes sobre por qué las partículas se mueven o no. En el mismo sentido nuestros instrumentos de recolección de datos no nos permiten incluir la dimensión Propiedades ya que no hemos entrado a analizar el tipo de justificaciones que hacen los alumnos de las propiedades de las sustancias. Por último, hemos incluido una nueva dimensión 
Diversidad que surge de la relevancia otorgada a la distinción entre los tipos de partículas que forman las sustancias (moléculas, iones y átomos).

En resumen, algunas de estas dimensiones y categorías que provienen de estudios de investigación anteriores han sido de gran utilidad para diseñar una nueva herramienta analítica para describir y analizar el conocimiento de estudiantes de $4^{\circ}$ de ESO sobre la materia en un nivel avanzado.

\section{Metodología}

\section{Contexto y muestra}

El instituto de secundaria obligatoria donde llevamos a cabo nuestro estudio se encuentra en un área metropolitana industrial cerca de Barcelona. En esta primera parte del estudio participaron dieciocho estudiantes de $4^{\circ}$ de ESO. Estos estudiantes ya habían optado por estudiar Física y Química como asignatura optativa y, por lo tanto, se les considera estudiantes que tienen algún interés por las ciencias. Estos estudiantes participaron en la implementación de una SEA diseñada por las propias autoras titulada «La materia por dentro». Así esta SEA considera los siguientes principios básicos:

Modelización: se promueve la modelización en la clase de ciencias incorporando actividades en el aula en las que se les pide a los estudiantes: expresar sus ideas iniciales sobre un hecho o fenómeno cotidiano, evaluarlas y revisarlas en función del análisis e interpretación de datos o como resultado de la discusión o contraste de diferentes ideas entre pares, estructurarlos para acordar un modelo final y aplicarlo en otras situaciones o fenómeno.

Conocimiento del contenido esencial: en toda la SEA solo se ha abordado el conocimiento de contenido necesario y suficiente para desarrollar un modelo conceptual de la materia.

Sentido de propósito relacionado con el contenido: la estructura de la SEA diseñada se ha orientado utilizando preguntas clave de conducción, que contribuyen a problematizar ideas.

Conexiones de fenómenos cotidianos: la SEA se refiere constantemente a situaciones cotidianas como una forma de acercarlas a la vida cotidiana de los estudiantes.

Sostenibilidad: las tareas y experiencias propuestas son asequibles tanto en tiempo como en costos para facilitar su adaptación/adopción en otros contextos.

Lenguaje simbólico: la mayoría de las tareas enfocan la demanda en representaciones simbólicas, gráficas o visuales del mundo submicroscópico.

\section{Recopilación de datos}

Los datos recopilados son las producciones de los estudiantes en algunas de las actividades realizadas durante la implementación de la SEA. Los enunciados de la actividades seleccionadas se muestran en la sección de resultados y consisten en preguntas abiertas de tipo descriptivo e interpretativo. La implementación tuvo lugar durante el primer trimestre del curso 2016-2017 a lo largo de 6 semanas, en sesiones de una hora, tres días a la semana, es decir, en total se recopilaron las producciones de los estudiantes en algunas de las actividades realizadas a lo largo de 18 horas. Estas actividades cumplieron el doble propósito de contribuir al desarrollo de las ideas de los estudiantes y a la recopilación de datos de investigación. Todas estas actividades fueron realizadas en la lengua vehicular de enseñanza (catalán) y han sido traducidas al castellano junto con las respuestas de los alumnos para este artículo. Esta traducción ha sido llevada a cabo por las investigadoras de manera que se asegura la correspondencia de las ideas en las dos lenguas. 
Los estudiantes entregaron todas las actividades que completaron en clase en formato digital a través de una plataforma electrónica (Moodle) tan pronto como terminaron.

Después de cada sesión, la primera autora de este artículo, que también era la profesora de ciencias del grupo de estudiantes, descargó todas las actividades y las codificó manualmente asignando un nuevo nombre a cada estudiante para mantener su anonimato.

Durante la implementación de la SEA esta profesora escribió los comentarios de los estudiantes sobre ciertas tareas en un diario de campo, así como sus intervenciones orales para la resolución de dudas relacionadas con el análisis de datos cuando fue necesario. En algunas ocasiones, cuando las interpretaciones podían resultar confusas, se pedían aclaraciones a los alumnos sobre sus producciones, a través del correo electrónico. Se procuró que estas intervenciones fueran sencillas y escuetas para no influir en la respuesta de los estudiantes.

Para mantener los estándares éticos de la investigación se obtuvo el permiso de las familias para la utilización de los datos en la investigación mediante una autorización que ha quedado archivada en el centro escolar y cuya existencia ha quedado certificada mediante un documento específico firmado por el director del centro.

\section{Análisis de los datos}

El análisis de datos se realizó utilizando una metodología de investigación cualitativa dentro del paradigma interpretativo que permite investigar las diferentes concepciones de los estudiantes sobre una situación o fenómeno específico (Marton 1981). El objetivo de este estudio es construir un conjunto de categorías relacionadas y agrupadas por dimensiones que permitan el análisis cualitativo de los datos relacionados con el conocimiento de la materia por parte de los estudiantes.

Para la construcción de estas categorías, se analizaron las producciones de los estudiantes en actividades seleccionadas de la SEA.

El proceso de análisis de estos datos es un proceso inductivo-deductivo de categorías que permite agruparlas en dimensiones. Para el análisis de los datos de entrada se hizo un análisis exploratorio por actividad, dentro de las elegidas, como se ha señalado anteriormente. Para cada una de las respuestas a las actividades se hizo una descripción detallada de la respuesta para ver qué elementos usaban los estudiantes tanto de tipo verbal como de representaciones.

Posteriormente, en un segundo análisis por actividad se intentaron encontrar elementos comunes para poder agrupar las respuestas. Es decir, se leyeron detenidamente las respuestas de los estudiantes intentando interpretar cuáles podrían ser sus ideas subyacentes cuando no corresponden a las de la ciencia. Tales ideas se agruparon en categorías. Dadas las concepciones de los alumnos sobre la materia que la literatura nos describe, estas categorías se compararon con las categorías descritas por diversos autores. Algunas de las categorías de las que informa la literatura fueron utilizadas tal como estaban descritas ya que nuestros datos encajaban en ellas, otras se reformularon para poder incluir algunas de las concepciones expresadas por nuestros estudiantes. Sin embargo, habíamos identificado algunas ideas que no podían ser incluidas en ninguna de las categorías que la literatura proporciona. Por ello, fue necesario definir una nueva dimensión que contiene algunas nuevas categorías y así recoger toda la variedad de ideas expresadas por los estudiantes. Así nació la dimensión Diversidad que glosaremos seguidamente. En la dimensión Diversidad aparecieron tres categorías, alumnos que no distinguían entre los diferentes tipos de partículas, alumnos que distinguían parcialmente el tipo de partículas -algunos sólo identificaban las moléculas y los iones, otros identificaban solamente las moléculas y los átomos y otros los átomos y los iones pero no las moléculas y los que distinguían completamente los diferentes tipos de partículas. 
Se utilizó la triangulación o verificación cruzada de la asignación de categorías por parte de las tres autoras para facilitar la validación del análisis de datos. Este proceso de triangulación asegura la fiabilidad del análisis como corresponde a un estudio cualitativo. También se utilizaron los datos recogidos a través del diario de campo como elemento para reforzar o refutar una determinada interpretación durante el análisis de los datos con el fin de aportar una mayor validez a los resultados.

\section{Resultados y discusión}

El análisis de las respuestas de los estudiantes a las actividades seleccionadas de la SEA diseñada se presenta a continuación, estructurado en torno a las cuatro dimensiones presentadas anteriormente (Conformación, Dinámica, Interacciones, Diversidad) y sus categorías correspondientes. Los ejemplos de las respuestas de los estudiantes (verbales o visuales) se utilizan para ilustrar cada una de las categorías en cada dimensión.

\section{Dimensión Conformación}

Esta dimensión está relacionada con las concepciones de los estudiantes acerca de la continuidad o discontinuidad de la materia, ignorando o asumiendo la existencia de partículas submicroscópicas. Dependiendo de estas concepciones de la naturaleza corpuscular de la materia, se pueden establecer las siguientes categorías:

Continuidad (C): la sustancia no está hecha de partículas.

Ninguno de los estudiantes que forma parte de nuestra muestra presenta esta concepción.

Incrustada (I) (partículas + sustancia): las partículas están incrustadas dentro de la sustancia.

Ninguno de los estudiantes de este estudio presentó esta concepción.

Las dos categorías anteriores de esta dimensión podrían interpretarse como una concepción preliminar de la materia que se ha superado principalmente en este nivel educativo $\left(4^{\circ}\right.$ de ESO). Después de una instrucción previa sobre la corpuscularidad de la materia, todos los estudiantes podían visualizar la materia como formada por partículas separadas, aunque no está claro cómo son estas partículas, como lo ilustran las siguientes categorías:

Granularidad (GR): (partículas + aire): las partículas están separadas por aire.

Un ejemplo de esta categoría es la respuesta de Marta a la pregunta 3b:

¿Cómo puedes explicar el comportamiento de diferentes sustancias (metálica, agua y aire) cuando se colocan dentro de una jeringa y se presiona empujando el émbolo?

«En la jeringa donde bay un pequeño cilindro de una sustancia metálica, podemos considerar que a nivel macroscópico bay aire y el metal en sí, pero a nivel submicroscópico bay partículas que forman el metal que imagino separadas con espacios que sería vacio [...] en la jeringa con agua, podemos considerar que a nivel macroscópico bay agua y aire, pero a nivel submicroscópico creo que hay buecos de vacio entre las partículas de agua. Finalmente, en la jeringa con el aire, considero que a nivel macroscópico bay aire, pero a nivel submicroscópico el aire sería el vacio. Sin embargo, no tengo una conclusión clara».

Como se muestra, la alumna considera que la sustancia metálica, el agua y el aire están formados por partículas. Sin embargo, interpretamos que este estudiante asocia el aire con el vacío, como si el aire no estuviera formado por partículas.

Corpuscularidad (CR) (partículas + vacío): las partículas mantienen las propiedades de las sustancias, es decir, las partículas son trocitos de la sustancia y no partículas submicroscópicas.

Esta categoría puede ilustrarse con la respuesta de Nuria (Figura 1) a la pregunta 1d: 
¿Cómo te imaginas un cubito de hielo por dentro? ¿De qué está hecho? ¿Cuáles son las unidades constituyentes de un cubito de hielo? ¿Son todas iguales?



Para esta estudiante ${ }^{2}$ (Figura 1), el hielo está formado por moléculas de agua congeladas. El hecho de que ella asocie el término congelado a moléculas como partículas submicroscópicas puede interpretarse como una asociación de propiedades macroscópicas de la sustancia (estado de agregación) a partículas submicroscópicas.

Discontinuidad (D): (partículas submicroscópicas + vacío): las partículas que forman sustancias se conciben separadas por vacío y tienen propiedades diferentes de las propiedades de las sustancias.

La respuesta de Aniol a la pregunta 3b es un ejemplo de esta categoría:

«En la jeringa con aire, puedes [empujar el pistón] pero sólo un poco. Eso es porque al ser un gas, tiende a ocupar el mayor lugar posible y cuando presionas el pistón, haces que las particulas se junten para que los espacios vacios entre ellas desaparezcan.»

La respuesta de este estudiante es un ejemplo de la conceptualización de sustancias (en este caso, aire) formadas por partículas separadas por vacío y la distancia entre partículas puede variar según las fuerzas o presiones externas.

\section{Dimensión Dinámica}

Esta dimensión está relacionada con el movimiento continuo de las partículas que forman la materia. Se pueden distinguir dos categorías:

Sin movimiento (SM): las partículas están en reposo.

La respuesta de Lía a la pregunta 5 h ilustra la conceptualización de los estudiantes descrita en esta categoría:

En el siguiente vídeo se puede ver un vaso de agua en cuyo interior se ha puesto una gota de colorante. Observad qué ocurre, ¿podríais explicar por qué se produce este hecho? Cómo explicaríais qué pasa a nivel submicroscópico, por qué se produce este hecho?

«Las particulas de colorante se mezclan con las de agua, es decir, podriamos encontrar, por ejemplo, una secuencia que sería una partícula de agua, una de colorante y otra de agua.»

La respuesta de la alumna hace referencia a la disposición de las partículas para explicar el fenómeno pero no habla de como se llega a esta distribución.

Movimiento (M): las partículas están en movimiento continuo.

La respuesta de Max a la pregunta 5h ilustra la conceptualización de los estudiantes descrita en esta categoría:

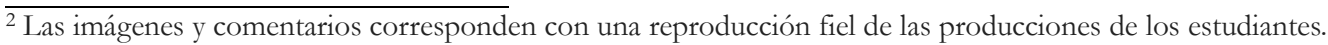


"Como las partículas se van moviendo libremente por todo el espacio que pueden, llega un momento que las particulas rojas ocupan todo el vaso y por eso se ve rojo».

\section{Dimensión Interacciones}

Esta dimensión se refiere a las concepciones de los estudiantes sobre las uniones o enlaces entre las partículas que forman las sustancias. A partir de los datos analizados, se pueden establecer las siguientes categorías:

Particulas sueltas (PS): las partículas no están unidas.

Un ejemplo de esta categoría es la respuesta de Aniol a la pregunta 5a:

A continuación, proponemos una representación submicroscópica de tres sustancias: las partículas de agua se representan con cuadrados, las partículas de aceite con triángulos y las partículas de etanol con círculos. ¿Las partículas de cada sustancia están unidas entre sí? ¿Cómo representarías los enlaces entre ellas en la imagen?

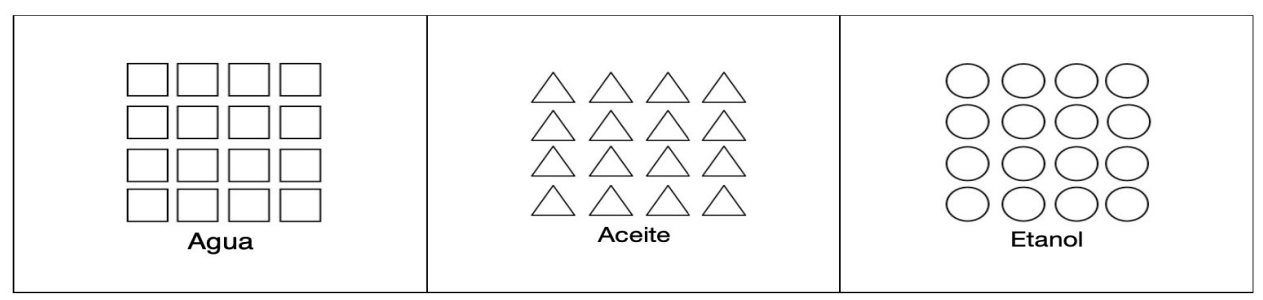

«No. Las uniones se producirian porque el agua, por ejemplo, está en un recipiente. Estas uniones serían vacio, ya que entre partícula y partícula no bay nada.»

Este estudiante interpreta que lo que hace que las partículas de la sustancia líquida estén unidas es el recipiente que las contiene y no hay uniones entre partículas, sólo vacío.

Particulas unidas en algunas ocasiones (PUAO): las partículas están unidas sólo en estado sólido.

Para mostrar esta categoría, usamos la respuesta de Pep a la pregunta 1e:

¿Cómo puedes imaginar las partículas (átomos, moléculas, iones) que forman el agua, el aire, el azúcar? Haz un dibujo usando símbolos y agregando etiquetas y descripciones.

En el dibujo de Pep (Figura 2), solo se representan las uniones entre las partículas que forman azúcar (es decir, una sustancia en estado sólido) y las partículas están distantes entre sí. Para el agua (es decir, una sustancia en estado líquido), el alumno representaba sus partículas muy cerca una de la otra pero sin ningún vínculo explícito. En el caso del aire (es decir, sustancia en estado gaseoso), sus partículas se representan muy separadas entre sí sin uniones entre ellas. Por lo tanto, según esta concepción, sólo las partículas de les sustancias en estado sólido están unidas entre sí.

Partículas unidas sin distinguir las uniones intermoleculares de las uniones interatómicas (PUSD)

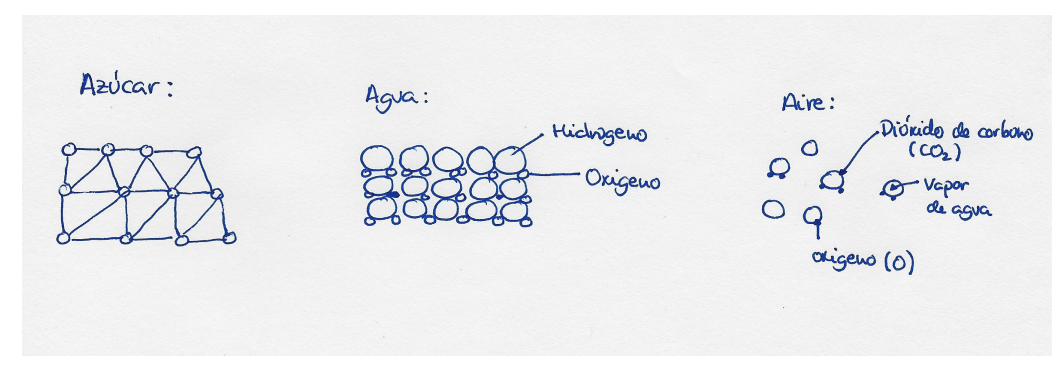

Figura 2. Ejemplo categoría PUAO (Pep) 
Un ejemplo que ilustra esta categoría es la representación de Aida (Figura 3) al responder a la pregunta 17a:

Haz una representación de cómo imaginas la sustancia agua en los tres estados de la materia. Piensa en el agua $\left(\mathrm{H}_{2} \mathrm{O}\right)$ cuando está en estado sólido (hielo) a $-10^{\circ} \mathrm{C}$, en estado líquido (agua) a $20^{\circ} \mathrm{C}$ o en estado gaseoso (vapor de agua) a $110^{\circ} \mathrm{C}$. Usa diferentes símbolos para representar los átomos de oxígeno $(\mathrm{O})$ y los átomos de hidrógeno $(\mathrm{H})$, y también representa las uniones entre partículas de acuerdo con su fuerza (por ejemplo, más gruesas o más delgadas, líneas continuas o discontinuas, diferentes colores, etc.). Explica todo lo que consideres necesario para que se pueda comprender tu representación de la sustancia a nivel submicroscópico.

En el dibujo de Aida (Figura 3), podemos observar que las moléculas se identifican como las partículas que forman el agua en estado líquido. Estas moléculas (dibuja hasta tres) están representadas con círculos de líneas discontinuas amarillas dentro de las cuales hay tres puntos, uno negro que representa átomos de oxígeno y dos grises que representan átomos de hidrógeno, que están unidos entre sí con uniones débiles representados con líneas discontinuas azules. Sin embargo, las uniones entre las moléculas no están representadas.

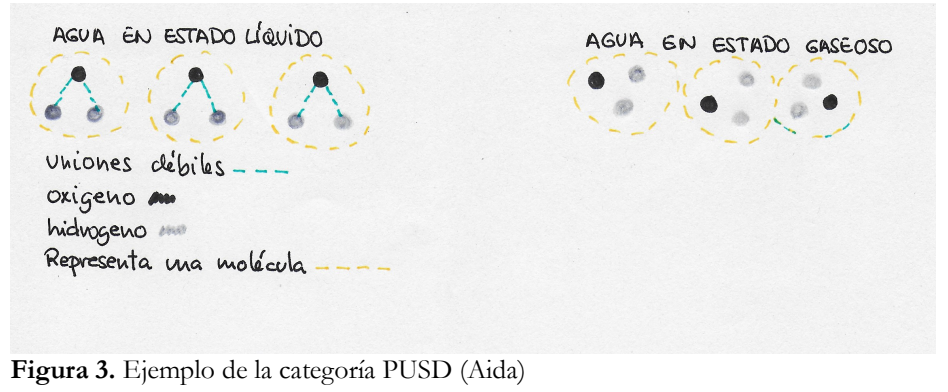

Si comparamos sus dibujos para el agua en estado líquido y en estado gaseoso, podemos ver cómo desaparecen las uniones débiles representadas antes con líneas discontinuas azules. Estas líneas representan uniones entre los átomos de oxígeno e hidrógeno. Por lo tanto, podemos interpretar que esta estudiante no distingue las uniones entre moléculas (uniones intermoleculares) de las uniones entre átomos (uniones interatómicas).

Particulas unidas distinguiendo las uniones intermoleculares de las uniones interatómicas (PUDU): uniones más débiles entre moléculas (uniones intermoleculares), uniones más fuertes entre átomos o iones (uniones interatómicas).

Un ejemplo de esta categoría es la representación de Zoe (Figura 4) que corresponde a su respuesta a la pregunta $15 \mathrm{~b}$ :

Haz una representación de cómo imaginas las siguientes sustancias en los tres estados de la materia y escribe lo que consideres necesario para explicar cómo las imaginas a un nivel submicroscópico.

AZUFRE: Piensa en el azufre $\left(\mathrm{S}_{8}\right)$ cuando está en estado sólido por debajo de $115^{\circ} \mathrm{C}$ (punto de fusión), en estado líquido (entre $115^{\circ} \mathrm{C}$ y $444^{\circ} \mathrm{C}$ ) y en estado gaseoso por encima de 444 ${ }^{\circ} \mathrm{C}$ (punto de ebullición).

ALUMINIO: Considera el aluminio ( $\mathrm{Al}$ ) a temperatura ambiente en estado sólido y después fundido a partir de aproximadamente $660^{\circ} \mathrm{C}$.

CLORURO DE POTASIO: considera el cloruro de potasio $(\mathrm{KCl})$ a temperatura ambiente en estado sólido, después de fundir a aproximadamente $776^{\circ} \mathrm{C}$ y después de hervir a $1477^{\circ} \mathrm{C}$. 




ordencoles y urides ento elles Leyenda:

$O=$ molécula de cazutue

(5) = átomo de azutre
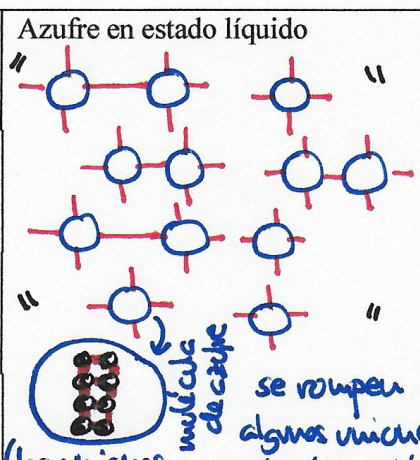

(laschicuos eutréclowes siguer igveles)

" "

= movimiento continuo

algurs vinicues eutre las undicells $y$ estan un poco desurcle wodes

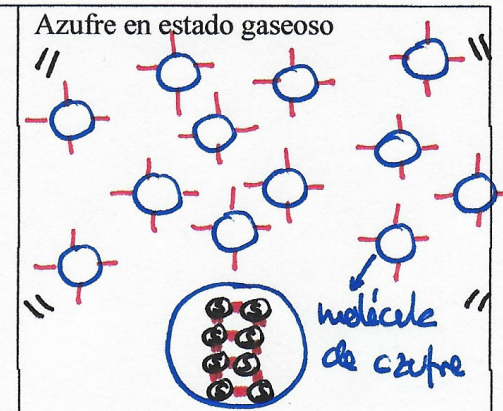

se wupen todes los mians entre los molícules y estar esparcides por todo el espacio (las cuicus outue d́bunos siguen igueles )

- surión débil

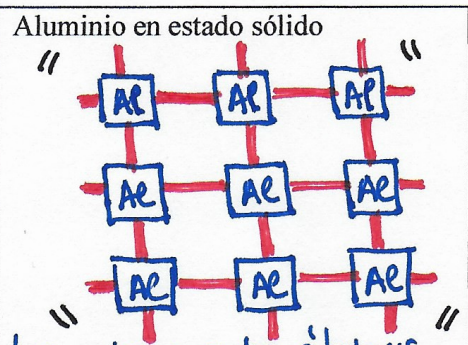

Las mioves entre áfolmos de alucunio sow treetis y estan todos orclenodos

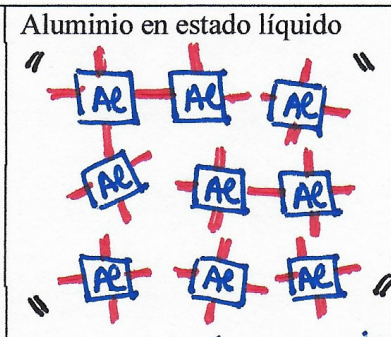

Se volupen algues mianes y estan un poco desordenedes
Aluminio en estado gaseoso thet

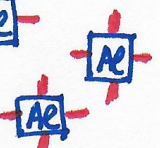

$\checkmark A R$ Se voupen bolos los vicuers y estar esparcicles por fodo de espeno Leyenda:

$$
A l=\text { átumo de aluminio = unión fuerte }
$$

" " = movimiento continvo

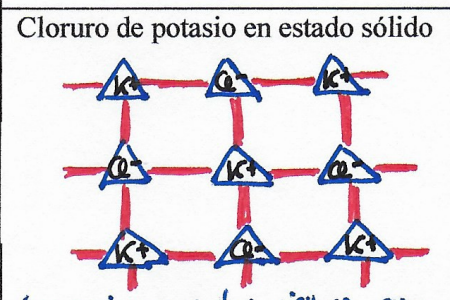

los cuicuses entre iones son


tuertes. Estan tocles cle formo ovde-


Kt=ión putesio $/ \mathrm{Ce}^{-}=$ión clowr

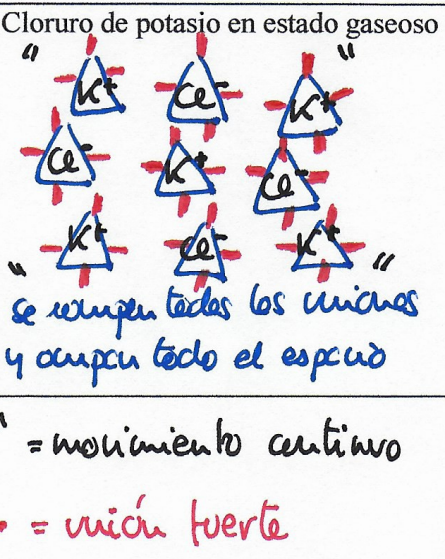

Figura 4. Ejemplo de categoría PUDU (Zoe)

En la figura 4, podemos observar que Zoe distingue entre diferentes uniones en términos de su fuerza: uniones más débiles entre moléculas que forman la sustancia azufre, uniones más fuertes entre átomos de azufre que forman moléculas de azufre, uniones más fuertes entre átomos metálicos que forman la sustancia aluminio y uniones más fuertes entre iones que 
forman cloruro de potasio como sustancia. Por lo tanto, Zoe representa la sustancia de azufre en estado sólido como la unión de las moléculas de azufre (círculos azules) con delgadas líneas rojas que indican que estas uniones son más débiles. Cada molécula de azufre se representa como formada por la unión fuerte (indicada por líneas gruesas de color rojo) de ocho átomos de azufre que están representados por círculos negros dentro de los cuales está la letra $\mathrm{S}$ que se corresponde al símbolo de azufre. Por otro lado, en la representación de la sustancia en el estado líquido, no todas las moléculas están unidas, aunque los enlaces entre las moléculas de azufre tienen la misma línea que en la representación de la sustancia en el estado sólido. Esto puede interpretarse como si la fuerza de los enlaces entre las moléculas de la sustancia en estado sólido y líquido sea la misma. Entonces, la diferencia entre la sustancia en estado sólido y líquido tiene que ver con el número de enlaces entre las moléculas, de modo que en el estado sólido todas las moléculas están unidas entre sí, mientras que en el estado líquido solo algunas moléculas están unidas entre sí. En el caso de la sustancia en estado gaseoso, ninguna de las moléculas estaría vinculada a ninguna otra, según la representación de la alumna. En relación a la sustancia aluminio, Zoe la representó en estado sólido en términos de la unión de átomos de aluminio. Los átomos de aluminio se representan con un cuadrado dentro del cual está el símbolo Al. Los enlaces entre los átomos de aluminio se representan con una línea gruesa que indica que los enlaces son fuertes. En el caso del estado líquido, el grosor de las líneas entre los átomos permanece, aunque no todos los átomos están unidos entre sí. En el caso del estado gaseoso, ninguno de los átomos está representado como unido. Finalmente, Zoe representó la sustancia cloruro de potasio mediante la unión de iones de potasio (representados por triángulos dentro de los cuales aparece el símbolo $\mathrm{K}+$ ) e iones de cloruro (representados por triángulos dentro de los cuales aparece el símbolo $\mathrm{Cl}$-). Las líneas que unen los diferentes triángulos son más gruesas, lo que indica que las uniones entre iones son fuertes. Los triángulos que representan iones de potasio e iones de cloruro se alternan en filas y columnas para que cada ión de cloruro esté rodeado de iones de potasio y viceversa. En el caso del estado líquido, no todos los iones están unidos, pero el grosor de las líneas entre los que están unidos es el mismo que para el estado sólido. En el caso del estado gaseoso, ninguno de los iones está unido. Podemos darnos cuenta de que las representaciones de los estudiantes condensan detalles muy específicos sobre su modelo de unión y fuerza entre partículas.

\section{Dimensión Diversidad}

Esta dimensión tiene como objetivo describir el conjunto de concepciones que los estudiantes tienen sobre la naturaleza de las partículas individuales que forman sustancias. El surgimiento de categorías bajo esta dimensión muestra que algunos estudiantes presentan una concepción de la materia consistente con el modelo conceptual avanzado de la materia, mientras que otros no. Para la ilustración de las diferentes categorías en esta dimensión, se han utilizado las representaciones hechas por los estudiantes para responder a la pregunta 15b expuesta anteriormente.

No diferenciación de particulas (NDP): No distinción entre moléculas, iones y átomos.

La representación de Dafne (Figura 5) en respuesta a las preguntas de la actividad 15b es un ejemplo de esta categoría: 


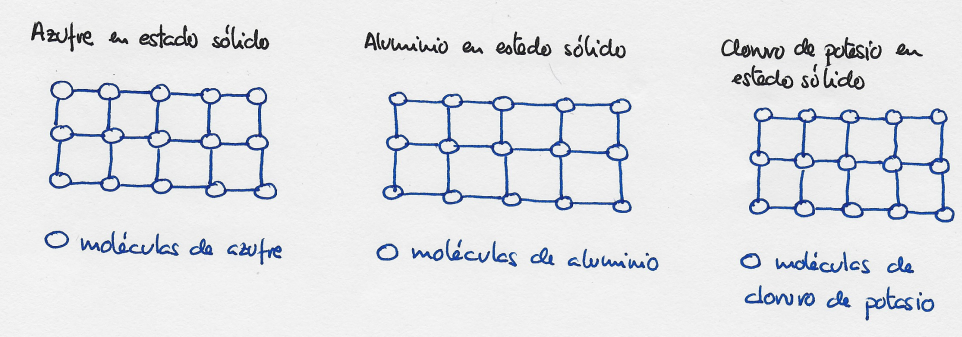

Figura 5. Ejemplo de la categoría NDP (Dafne)

Para esta estudiante, las partículas que forman las tres sustancias son todas moléculas. Ella no proporciona ninguna información sobre la composición de cada una de estas «moléculas» de azufre, aluminio y cloruro de potasio.

Diferenciación parcial de partículas (PD): algunas de las partículas (moléculas, iones o átomos) se distinguen, pero no los tres tipos.

Esta categoría puede ilustrarse con la respuesta de Carlota (Figura 6) en respuesta a las preguntas de la actividad 15b. Podemos observar que para el cloruro de potasio, la alumna dibuja triángulos dentro de los cuales se escriben los símbolos $\mathrm{K}+$ y $\mathrm{Cl}$-, que representan iones (positivo y negativo). En el caso del azufre, ella usa cuadrados en cuyo interior está el símbolo $\mathrm{S}$, que representa la molécula de azufre. Sin embargo, cuando representa la sustancia de aluminio, usa círculos dentro de los cuales aparece el símbolo $\mathrm{Al}$, lo que indica que representan «moléculas de aluminio». Por lo tanto, podemos interpretar que considera que el cloruro de potasio está formado por iones de cloruro y potasio, mientras que el azufre y el aluminio están formados por moléculas. Es decir, ella no distingue entre moléculas (grupos de átomos no metálicos) y átomos metálicos. Vale la pena mencionar que durante la SEA esta definición operativa de moléculas como grupos de átomos no metálicos se introdujo y desarrolló a lo largo de varias tareas para permitir a los estudiantes interpretar si una sustancia está formada por moléculas al identificar si los átomos que forman parte de estas moléculas son metales o no metales según su clasificación en la tabla periódica. 


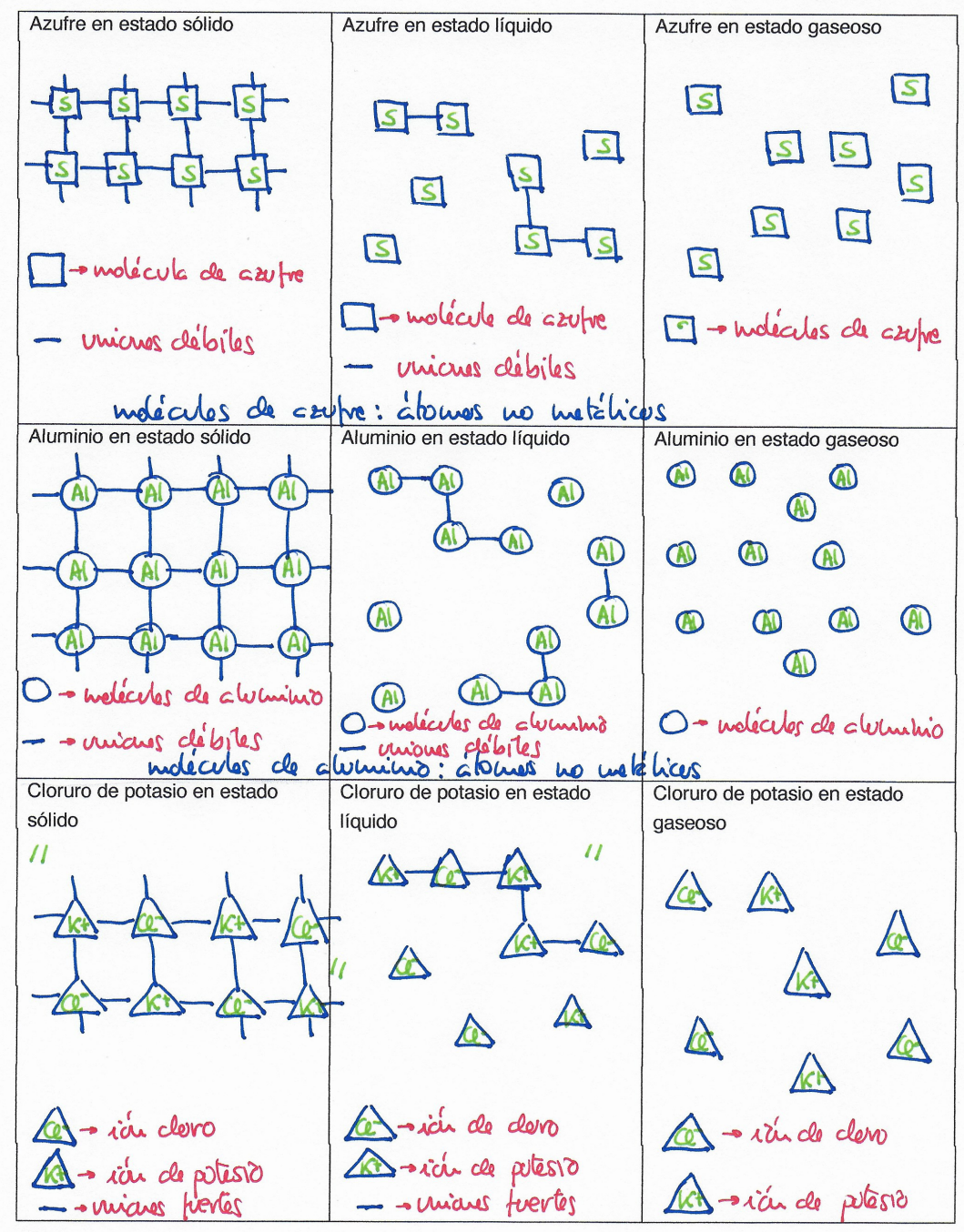

Figura 6. Ejemplo de categoría DP (Carlota)

Diferenciación total de particulas (DT): las partículas se diferencian adecuadamente entre iones, moléculas y átomos metálicos.

Un ejemplo de esta categoría son las representaciones dibujadas por Lara (Figura 7) en respuesta a las preguntas de la actividad $15 \mathrm{~b}$.

En esta representación se puede observar que Lara distingue los tres tipos diferentes de partículas que forman las tres sustancias: moléculas de azufre (representadas por rectángulos verdes), átomos de aluminio (representados por círculos grises) e iones de potasio (representados por círculos naranjas) y de cloro (representados por círculos púrpuras) para cloruro de potasio. La representación de los tres estados de la materia para cada sustancia nos permite interpretar que la alumna tiene una idea de la partícula que permanece a pesar de las transformaciones que ocurren en los cambios de estado. Por lo tanto, representa que las uniones que se rompen cuando la sustancia azufre cambia del estado sólido al líquido y del estado líquido al gaseoso son las uniones entre moléculas (intermoleculares) y, por lo tanto, la partícula-molécula se conserva. En el caso de la sustancia aluminio, las uniones entre los átomos de aluminio se rompen y se conservan los átomos metálicos (aluminio). Del mismo modo, en el caso del cloruro de potasio, las uniones entre los iones de cloro y de potasio también se rompen, pero los iones (cloro y potasio) se conservan. Esta estudiante también 
indica que las partículas que forman estas sustancias están en movimiento continuo usando los símbolos ""'.



Figura 7. Ejemplo de la categoría DT (Lara)

\section{Conclusiones}

En primer lugar, este estudio de investigación ha permitido caracterizar las concepciones de los estudiantes de $4^{\circ}$ de ESO sobre la materia. Nuestros resultados han mostrado la prevalencia de las concepciones de ciertos estudiantes ya informadas en la literatura (Harrison y Treagust 2002, Albanese y Vicentinni 1997, Griffiths y Preston 1992, Ben-Zvi, Eylon y Silberstein 1986). Además, este estudio muestra evidencia de la presencia de otras concepciones relacionadas con la dimensión específica acuñada como Diversidad de partículas (moléculas, átomos e iones), relacionada con la construcción del llamado modelo conceptual de la materia. En segundo lugar, el estudio también ha demostrado la utilidad de las representaciones visuales hechas por los estudiantes, como complemento de sus respuestas verbales para ayudar a interpretar sus concepciones, ya que estos dibujos presentan detalles 
ricos. Así, por ejemplo, los estudiantes representan fácilmente la fuerza de las uniones entre diferentes tipos de partículas con líneas de diferentes colores o con diferentes grosores. También han representado fácilmente los diferentes tipos de partículas que forman una sustancia específica a través de símbolos (círculos, triángulos, cuadrados con letras dentro) junto con especificaciones verbales, que reflejan sus concepciones subyacentes. A menudo, el uso de terminología científica (por ejemplo, moléculas, átomos metálicos, iones, etc.) por parte de los estudiantes carece de significado para ellos o significa algo diferente del significado científico (por ejemplo, los estudiantes usan el término molécula cuando hablan de átomos). Algunas de las representaciones visuales también proporcionaron detalles como la idea del movimiento continuo de partículas, mediante el uso de comillas en los tres estados de la materia. Por lo tanto, nuestros resultados respaldan las afirmaciones de que las visualizaciones son una forma muy útil de acceder a las concepciones de los estudiantes (Cheng 2018, Cheng y Gilbert 2017 y 2009, Adadan, Irving y Trundle 2009).

\section{Una herramienta analítica para explorar las concepciones sobre la materia de los estudiantes}

Como sugiere nuestra pregunta de investigación, este estudio también tenía como objetivo construir una herramienta analítica que facilite la caracterización de las concepciones sobre la materia de los estudiantes de secundaria en relación con un modelo avanzado de la materia. Hemos podido elaborar esta herramienta en torno a cuatro dimensiones diferentes: Conformación, Dinámica, Interacciones y Diversidad, que se han descrito a través de un conjunto de categorías. La tabla 2 muestra el instrumento analítico que se ha construido como producto de este estudio de investigación.

Tabla 2. Dimensiones y categorías del instrumento de análisis para explorar las concepciones de los estudiantes en relación a un modelo avanzado de materia

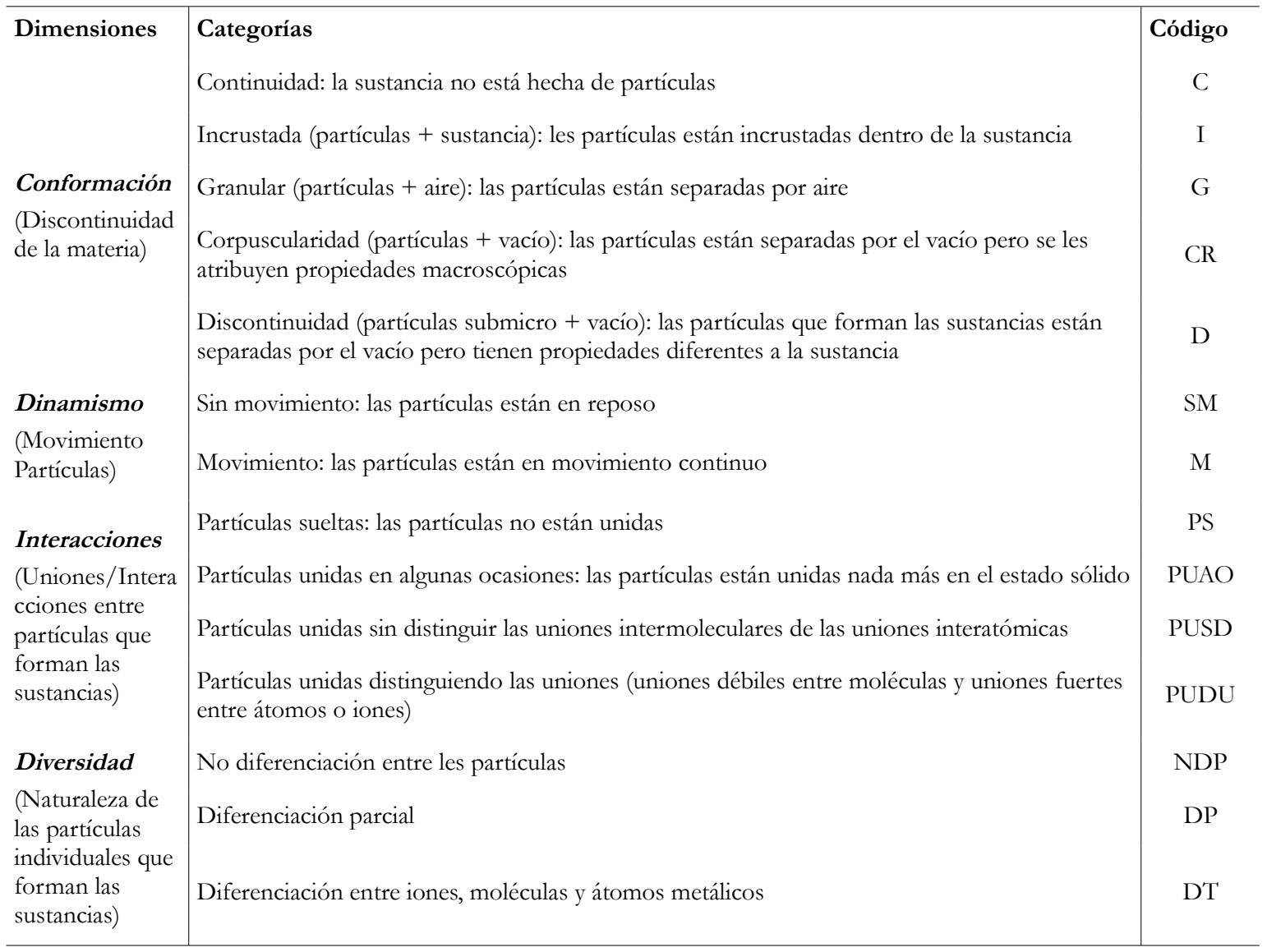


Como se informó en páginas anteriores, algunas de estas dimensiones están adaptadas de la literatura del dominio que describe las conceptualizaciones de los estudiantes en torno a las ideas clave relacionadas con un modelo conceptual avanzado de la materia. Como señalamos antes, este modelo avanzado de materia distingue entre partículas submicroscópicas (moléculas, átomos e iones) para interpretar las propiedades y/o cambios de las sustancias. En ese sentido, la dimensión Diversidad es nueva y complementa otros estudios como el propuesto por Talanquer (2009). Dentro de esta dimensión, hemos construido varias categorías que emergen del análisis de los dibujos de los estudiantes en sus representaciones de la materia a nivel submicroscópico. Esta herramienta analítica puede ayudar a caracterizar los modelos de materia de los estudiantes de manera sistemática, cuando se les pide que susciten y apliquen sus ideas a diferentes situaciones relacionadas con la interpretación de las propiedades de las sustancias o fenómenos de transformación de la materia. El proyecto de investigación en curso de las autoras también ha demostrado que esta herramienta analítica es útil para estudiar la evolución de los modelos de materia de los estudiantes a lo largo de la instrucción.

\section{Referencias}

Adadan, E., Irving, K.E., Trundle, K.C. (2009). Impacts of Multi-representational Instruction on High School Students' Conceptual Understandings of the Particulates Nature of Matter. International Journal of Science Education, 31(13), 1743-1775.

Albanese, A., Vicentinni, M., (1997) Why do we believe that an atom is colourless? Reflections about the teaching of the particle model. Science and Education 6, 251-261

Andersson, B. (1990). Pupils' Conceptions of Matter and its Transformations (age 12-16). Studies in Science Education, 18, 53-85

Barke HD., Hazari A., Yitbarek S. (2009) Students' Misconceptions and How to Overcome Them. In Barke HD., Hazari A., Yitbarek S. (Eds), Misconceptions in Chemistry (pp.21-26), Springer, Berlin, Heidelberg.

Benarroch Benarroch, A. (2000a). El desarrollo cognoscitivo de los estudiantes en el área de la naturaleza corpuscular de la materia. Enseñanza de Las Ciencias, 18(2), 235-246.

Benarroch Benarroch, A. (2000b). Del modelo cinético-corpuscular a los modelos atómicos, Alambique, 23.

Ben-Zvi, R., Eylon, B., \& Silberstein, J. (1986). Is an atom of copper malleable? Journal of Chemical Education, 63, 64-66

Chang, R., (2002). Química (pp. 182 y 418) McGraw-Hill Interamericana Editores, S.A.

Cheng, M., Gilbert, J.K. (2009). Towards a Better Utilization of Diagrams in Research into the Use of Representative Levels in Chemical Education. In Gilbert, J.K. and Treagust, D. (Eds.), Multiple Representations in Chemical Education (pp. 55-73). Springer Science+ Bussiness Media B.V.

Cheng, M., Gilbert, J.K. (2017). Modelling students' visualisation of chemical reaction, International Journal of Science Education, 39(9), 1173-1193

Cheng, M. (2018). Students' visualisation of chemical reactions- insights into the particle model and atomic model. Chemistry Education Research and Practice, 19, 227-239

de Vos, W., Verdonk, A. H. (1996). The particulate nature of matter in science education and in science. Journal of Research in Science Teaching, 33, 657-664. 
Duit, R., Gropengießer, H., Kattmann, U. (2005). Towards science education research that is relevant for improving practice: the model of educational reconstruction. In Fischer, H.E. (Ed.) Developing Standards in Research on Science Education (pp. 1-9) London: Taylor and Francis

Griffiths, A.K., Preston, K.R. (1992). Grade-12 students' misconceptions relating to fundamental characteristics of atoms and molecules. Journal of Research in Science Teaching, 29, 611-628

Hadenfeldt, J. C., Liu, X., Neumann, K. (2014). Framing students' progression in understanding matter: a review of previous research. Studies in Science Education, 50(2), 181-208.

Harrison, A. G., Treagust, D. F. (2002). The particulate nature of matter: Challenges in understanding the submicroscopic world. In J. Gilbert, O. De Jong, R. Justi, D. F. Treagust, Van Driel Jan H. (Eds.), Chemical education: Towards research-based practice (pp. 189-212). Boston: Kluwer Academic Publisher.

Izquierdo-Aymerich, M. \& Adúriz-Bravo, A. (2003). Epistemological Foundations of School Science. Science Education 12, 27-43.

Johnson, P. (1998). Progression in children's understanding of a 'basic' particle theory: A longitudinal study. International Journal of Science Education, 20(4), 393-412.

Johnson, P., Tymms, P. (2011). The emergence of a learning progression in middle school chemistry. Journal of Research in Science Teaching, 48(8), 849-877.

Liu, X., Lesniak, K. (2005). Students' progression of understanding of the matter concept from elementary to high school. Science Education, 89, 433-450.

Liu, X., Lesniak, K. (2006). Progression in children's understanding of the matter concept from elementary to high school. Journal of Research in Science Teaching, 43(3), 320-347.

Marton, F. (1981). Phenomenography-describing conceptions of the world around us. Instructional Science, 10, (2), 177-200

Moltó. M.A., Pintó, R. (2013). L'ús del triplet de la química en la construcció del concepte dissolució a primer de batxillerat. Universitat Autònoma de Barcelona.

Renström, L., Andersson B., Marton, F. (1990). Students' Conceptions of Matter. Journal of Educational Psychology, 82(3), 555-569.

Smith, C. L., Wiser, M., Anderson, C. W., Krajcik, J. S. (2006). Implications of Research on Children's Learning for Standards and Assessment: A Proposed Learning Progression for Matter and the Atomic-Molecular Theory. Measurement: Interdisciplinary Research \& Perspective, 4(1-2), 1-98.

Stern, L., Ahlgren, A. (2002). Analysis of students' assessments in middle school curriculum materials: Aiming precisely at benchmarks and standards. Journal of Research in Science Teaching, 39(9), 889-910.

Stevens, S. Y., Delgado, C., Krajcik, J. S. (2010). Developing a hypothetical multi-dimensional learning progression for the nature of matter. Journal of Research in Science Teaching, 47(6), 687-715.

Talanquer, V. (2009). On Cognitive Constraints and Learning Progressions: The case of "structure of matter." International Journal of Science Education, 31(15), 2123-2136. 
Talanquer, V. (2018). Progression in reasoning about structure-property relationships. Chemistry Education Research and Practice. 19, 998-1009.

Wiser, M., Smith, C.L. (2008). Learning and Teaching about Matter in Grades K-8: What Should the Atomic- Molecular Theory be Introduced? In Vosniadou, S. (Ed.), International Handbook of Research on conceptual change (pp. 205-239). Routledge, New York. 